\title{
Comparison of Hemodynamic Response to Orotracheal Intubation with Conventional Laryngoscope versus Fiberoptic Bronchoscope
}

Khalid Zaeem Aslam, Sanum Kashif, Amna Gulrez

\begin{abstract}
Objective: To compare the hemodynamic response to orotracheal intubation via direct laryngoscope versus fiberoptic bronchoscope in patients undergoing general anesthesia.

Study Design: Comparative Study.

Place and Duration of Study: Anesthesia Department of Military Hospital, Rawalpindi from $1^{\text {st }}$ March to $30^{\text {th }}$ May 2014.

Materials and Methods: A total of 110 patients for elective surgeries with general anesthesia, were included and randomized into two groups. The patient in group $F(n=55)$ were intubated by direct laryngoscopy (DLS) and group L ( $n=55$ ) cases intubated by fiberoptic bronchoscopy (FOB). The hemodynamic response heart rate $(H R)$, systolic blood pressure (SBP), diastolic blood pressure (DBP) and mean arterial pressure (MAP) were recorded at baseline, at induction, at laryngoscopy, at the time of intubation and 3 minutes after intubation.

Results: The age of the patients ranged between 19-45 years. Average age of participants was 33.76 and 31.56 and average weight of patients was $71.22 \pm 1.493$ and $73.18 \pm 1.390 \mathrm{Kg}$ in group $F$ and L respectively. At induction, hemodynamic values dropped to $20 \%$ of the baseline values. At the time of intubation (laryngoscopy or fiberoptic bronchoscopy) and 3 min after intubation, hemodynamic values increased significantly $(p<0.05)$ in both groups.

Conclusion: Our study demonstrated that both groups (Fiberoptic bronchoscopy verses direct laryngoscopy) showed same hemodynamic responses during orotracheal intubations.
\end{abstract}

Key Words: Direct Laryngoscope (DLS), Fiberoptic Bronchoscope (FOB), Hemodynamic Response, Orotracheal Intubation.

How to cite this: Aslam KZ, Kashif S, Gulrez A. Comparison of Hemodynamic Response to Orotracheal Intubation with Conventional Laryngoscope versus Fiberoptic Bronchoscope. Life and Science. 2020; 1(2): 64-67. doi: https://doi.org/10.37185/LnS.1.1.56

\section{Introduction}

Hemodynamic responses are affected by the technique of laryngoscopy and the type of instruments like direct laryngoscope (DLS) and fiberoptic bronchoscope (FOB). ${ }^{1,2}$ These interventions increase sympathetic activity which results in hypertension and tachycardia.

Most routine orotracheal or nasotracheal intubations are performed with the help of conventional laryngoscope that has a curved or straight blade and other external adjuncts such as external laryngeal pressure, a bougie, a stylet, or Magill forceps may also be needed. ${ }^{3,4}$

FOB is an important instrument for difficult airway

Department of Anethesia

Military Hospital, Rawalpindi

Correspondence:

Maj Dr. Sanum Kashif

Department of Anethesia

Military Hospital, Rawalpindi

E-mail:sanumdr@gmail.com

Funding Source: NIL; Conflict of Interest: NIL

Received: Jul 15, 2019; Revised: Jan 28, 2020

Accepted: Feb 11, 2020

64 management. Fiberoptic intubation can avoid mechanical stimulus to oropharyngeal and laryngeal structures, thereby it is likely to attenuate hemodynamic responses during orotracheal intubation. However, relevant studies show conflicting results. ${ }^{5,6}$ The rationale of this study was to compare the hemodynamic response of orotracheal intubation using FOB versus DLS in patients undergoing general anesthesia.

\section{Materials and Methods}

The study was conducted at Anesthesia Department of Military Hospital, Rawal pindi from $1^{\text {st }}$ March to $30^{\text {th }}$ May 2014 after approval by ethical review committee of Military Hospital Rawalpindi and written informed consent, 110 adult patients, American Society of Anesthesiologists (ASA) physical status I-II, scheduled for elective surgeries under general anesthesia requiring orotracheal intubation were included in this study. Patients with anticipated difficult airways, asthma, cardiovascular disease, gastroesophageal reflux disease, morbid obesity and known hypertension, were excluded from the study. 
Patients were randomly divided equally into the $\mathrm{L}$ (laryngoscope) group $(n=55)$ and the $F$ (fiberoptic bronchoscope) group $(n=55)$. Randomization was based on computer-generated codes that were maintained in sequentially numbered opaque envelopes. All patients were nil by mouth (NPO) at the time of surgery. Baseline hemodynamics were recorded. Anesthesia was induced with Propofol $2 \mathrm{mg} / \mathrm{kg}$, Atracurium $0.5 \mathrm{mg} / \mathrm{kg}$ and Nalbuphine $0.1 \mathrm{mg} / \mathrm{kg}$ via $20 \mathrm{G}$ intravenous cannula. Hemodynamics were noted just after induction of anesthesia. Direct laryngoscopy or fiberoptic bronchoscopy was started as per randomization three minutes after Atracurium injection. In group L, the tracheal tube was inserted into the trachea under direct vision using a Macintosh laryngoscope (size 3.0) according to the conventional manner. In the group F, a FOB with an outer diameter of $5.1 \mathrm{~mm}$ was used for tracheal intubation. Hemodynamic responses were recorded at intubation and three minutes after intubation. After successful intubation, intermittent positive pressure ventilation (IPPV) was performed with a fresh gas flow of $1.5 \mathrm{~L} / \mathrm{min}$. Anesthesia was maintained with $1 \%$ isoflurane and $50 \%$ nitrous oxide in oxygen. Patients requiring more than one attempt to achieve successful intubation were excluded from the study. All data was analyzed with SPSS 10.1 statistical software (SPSS Inc., Chicago, USA). Demographic and clinical data from the two groups were compared using the two-tailed $t$-test and chi-square test. The comparisons of hemodynamic data of the two groups were made using the two-way repeated measure analysis of variance (ANOVA) and $t$-test. All quantitative data were expressed as mean standard deviation (SD). A $p<0.05$ was considered statistically significant.

\section{Results}

The study reported no significant difference in the demographic and baseline values of blood pressure (BP) and heart rate (HR) (Table 1). Age of the patients ranged between $19-45$ years. Average age of participants was 33.76 and 31.56 years $(p=0.815)$ as shown in (Figure 1 ) and average weight of patients was $71.22+\_1.493$ and $73.18+\_1.390(\mathrm{Kg})$ in group $F$ and $L(p=0.338)$ respectively (Figure 2$)$. At induction, haemodynamic values were decreased but within $20 \%$ of the baseline values. The baseline and induction values, at direct laryngoscopy and fiberoptic bronchoscopy, in both group $L$ and $F$ caused same significant increases in BP and HR $(p<0.05)$ (Table 1).

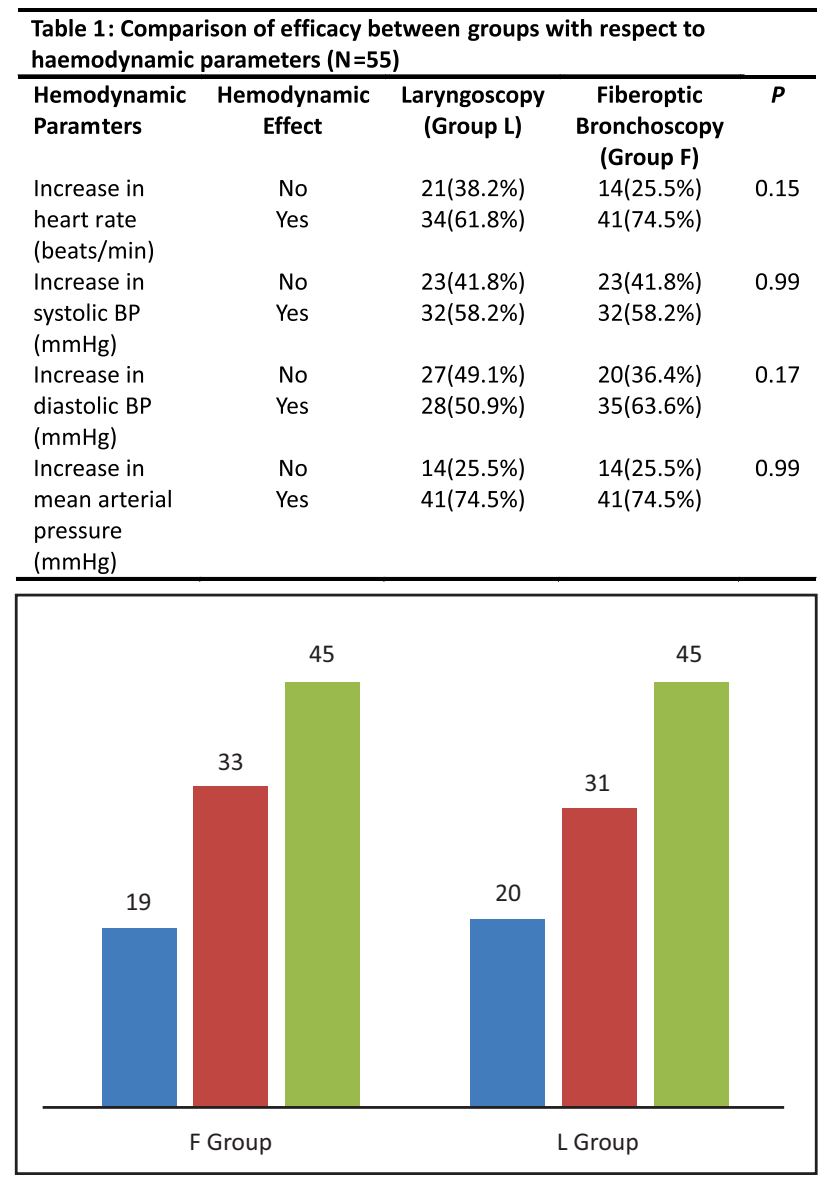

Fig 1: Median age in years between groups

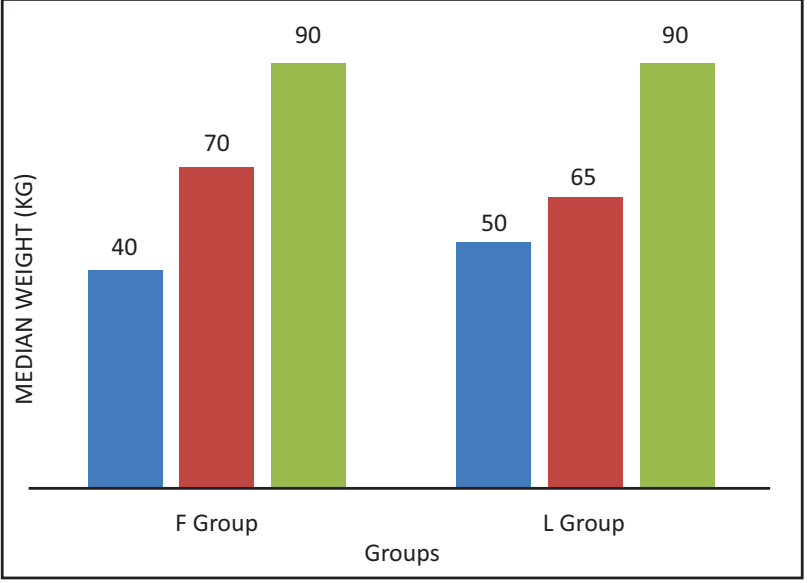

Fig 2: Mean weight in kg between groups

\section{Discussion}

Hemodynamic stability during airway manipulation for endotracheal intubation is crucial in surgeries 
under general anesthesia. Profound hemodynamic changes during direct laryngoscopy or fiberoptic bronchoscopy may cause deleterious effects. There are different ways to attenuate that hemodynamic response. ${ }^{7,8}$ Paul A et al, in their study on ASA I and II patients $(n=50)$ scheduled for elective surgery under general anesthesia requiring orotracheal intubation, demonstrated that the orotracheal intubations using different laryngoscopes produced similar hemodynamic responses. ${ }^{9}$ Micpovilyte $V$ et al showed that patients who were scheduled for different elective surgeries under general anesthesia, produced almost same stress response to tracheal intubation. ${ }^{10}$ Buhari FS, Selvaraj $\mathrm{V}$ et al, conducted a study on ASA I-II patients, $(n=90)$ requiring orotracheal intubation under general anethesia via different laryngoscopes. Patients were randomly allocated into three groups. Results of study showed that the orotracheal intubation via conventional laryngoscopy with Macintosh larnygoscope produced less haemodynamic response as compare to other laryngoscopes. ${ }^{11}$ Hegazy AA et al reported that endotracheal intubation using fiberoptic bronchoscope, produces more stimulus to the airway due to prolonged intubation time, which invalidates its benefit of avoiding oropharyngeal and laryngeal stimulation. ${ }^{12}$ In addition, the advancement of the tracheal tube over the FOB is often impeded when the Murphy's tip catches on the downward sagging epiglottis, arytenoid cartilage, vocal cords and anterior tracheal wall. On such occasions, the successful intubation often requires some specific maneuvers for example, rotating the tracheal tube, further lifting jaw upward and adjusting the patient's head-neck position which can result in hypertension and tachycardia. ${ }^{13}$ All these procedures are invasive, and may further stimulate the pharyngeal and laryngeal structures. These responses can be attenuated by different group of drugs e.g dexmedetomidine and fentanyl. ${ }^{14}$ During the fiberoptic intubation, the insertion cord must be placed into the trachea for guidance followed by advancing the tracheal tube over the insertion cord into the trachea and then the FOB is removed. ${ }^{15}$ This can cause repeated friction and irritation to the trachea. ${ }^{16,17}$ The laryngoscopy produces a balanced stimulation of vagal and cardiac accelerator fibers, whereas the intratracheal manipulation produces more sympathetic stimulation. Tracheal tube insertion itself is the most invasive stimulus and may be the major cause of cardiovascular responses to the tracheal intubation. ${ }^{18}$

\section{Conclusion}

Our study showed that orotracheal intubation with the help of FOB in surgeries under general anesthesia in adult patients, causes the same significant increase in hemodynamics as a DLS.

\section{REFERENCES}

1. Gill N, Purohit S, Kalra P, Lall T, Khare A. Comparison of hemodynamic responses to intubation: Flexible fiberoptic bronchoscope versus McCoy laryngoscope in presence of rigid cervical collar simulating cervical immobilization for traumatic cervical spine. Anesthesia, essays and researches. 2015; 9: 337-42.

2. Aggarwal H, Kaur S, Baghla N, Kaur S. Hemodynamic Response to Orotracheal Intubation: Comparison between Macintosh, McCoy, and C-MAC Video Laryngoscope. Anesthesia, essays and researches. 2019; 13: 308-12.

3. Zhang $Y$, Zeng Z, Xiao G, Zhang W, Lin W, Deng J. A randomized trial to evaluate a modified tracheal catheter with upper and lower balloons for anesthetic administration: effect on the cardiovascular, stress response, and comfort in patients undergoing laparoscopic cholecystectomy. BMC anesthesiology. 2019; 19: 211.

4. Bansal S, Bansal S, Kaur D, Sharma S. Comparative evaluation of intubating conditions and hemodynamic response to laryngoscopy and intubation with McCoy and Macintosh laryngoscopes; a prospective randomized study. Anaesthesia, Pain \& Intensive Care. 2018; 22: 193-8.

5. Zhang $Y$, Xiao D, Lin W, Zhang W, Luo R. Comparison of video laryngoscope, direct laryngoscopy and fibreoptic bronchoscope nasal intubation on hemodynamics, inflammatory and stress response. China Journal of Endoscopy. 2016; 22: 51-6.

6. Amini S, Shakib M. Hemodynamic changes following endotracheal intubation in patients undergoing cesarean section with general anesthesia: application of glidescope ${ }^{\circledR}$ videolaryngoscope versus direct laryngoscope. Anesthesiology and pain medicine. 2015; 5: e21836.

7. Sachidananda R, Umesh G. A review of hemodynamic response to the use of different types of laryngoscopes. Anaesthesia, Pain \& Intensive Care. 2019; 24: 201-8.

8. Rajan S, Kadapamannil D, Barua K, Tosh P, Paul J, Kumar L. Ease of intubation and hemodynamic responses to nasotracheal intubation using C-MAC videolaryngoscope with D blade: a comparison with use of traditional Macintosh laryngoscope. Journal of anaesthesiology, clinical pharmacology. 2018; 34:381-5.

9. Paul A, Nathroy A. Comparison of hemodynamic changes during laryngoscopy with McCoy and Macintosh laryngoscopes. Journal of Health Research and Reviews. 2017; 4:35-9. 
10. Micpovilytė V, Zubavičiūtè I, Rancevienè D, Macas A. Flexible fiberoptic intubation comparison with other intubation techniques. SVEIKATOS. 2018; 28: 75-83.

11. Buhari FS, Selvaraj V. Randomized controlled study comparing the hemodynamic response to laryngoscopy and endotracheal intubation with McCoy, Macintosh, and C-MAC laryngoscopes in adult patients. Journal of anaesthesiology, clinical pharmacology. 2016; 32: 505-9.

12. Hegazy AA, Al-Kawally $H$, Ismail EF, Abedlmabood MA, Mandour UA. Comparison between fiberoptic bronchoscope versus C-MAC video-laryngoscope for awake intubation in obese patients with predicted difficult airway. Research and Opinion in Anesthesia and Intensive Care. 2018; 5: 134-40.

13. Bharti N, Arora S, Panda NB. A comparison of McCoy, TruView, and Macintosh laryngoscopes for tracheal intubation in patients with immobilized cervical spine. Saudi journal of anaesthesia. 2014; 8: 188-92.

14. Mondal S, Ghosh S, Bhattacharya S, Choudhury B, Mallick S, Prasad A. Comparison between dexmedetomidine and fentanyl on intubation conditions during awake fiberoptic bronchoscopy: A randomized double-blind prospective study. Journal of anaesthesiology, clinical pharmacology. 2015; 31: 212-6.

15. Parasa M, Yallapragada SV, Vemuri NN, Shaik MS. Comparison of GlideScope video laryngoscope with Macintosh laryngoscope in adult patients undergoing elective surgical procedures. Anesthesia, essays and researches. 2016; 10: 245-9.

16. Hoshijima H, Denawa $Y$, Tominaga A, Shiga T, Nagasaka H. Videolaryngoscope versus Macintosh laryngoscope for tracheal intubation in adults with obesity: A systematic review and meta-analysis. Journal of clinical anesthesia. 2018; 44:69-75.

17. Tseng KY, Lu IC, Shen YC, Lin $\mathrm{CH}$, Chen PN, Cheng KI. A comparison of the video laryngoscopes with Macintosh laryngoscope for nasotracheal intubation. Asian journal of anesthesiology. 2017; 55: 17-21.

18. Godbole MR, Saundattikar G. A prospective randomized control trial-Comparison of hemodynamic response to laryngoscopy and intubation-Macintosh versus McCoy blade. Indian Journal of Clinical Anaesthesia. 2018; 5: 1103. 